task force on clinical practice guidelines. J Am Coll Cardiol. 2017;70: 252-89.

2. Jeong DS, Sung K, Kim WS, Lee YT, Yang JH, Jun TG, et al. Fate of functional tricuspid regurgitation in aortic stenosis after aortic valve replacement. J Thorac Cardiovasc Surg. 2014;148:1328-33.e1.

3. Zack CJ, Fender EA, Chandrashekar P, Reddy YNV, Bennett CE, Stulak JM, et al. National trends and outcomes in isolated tricuspid valve surgery. J Am Coll Cardiol. 2017;70:2953-60.

4. Kilic A, Saha-Chaudhuri P, Rankin JS, Conte JV. Trends and outcomes of tricuspid valve surgery in North America: an analysis of more than 50,000 patients from the Society of Thoracic Surgeons database. Ann Thorac Surg. 2013;96:1546-52.
5. Nath J, Foster E, Heidenreich PA. Impact of tricuspid regurgitation on long-term survival. J Am Coll Cardiol. 2004;43:405-9.

6. Chancellor WZ, Mehaffey H, Belleter JP, Hawkins RB, Speir AM, Quader MA, et al. Impact of tricuspid regurgitation with and without repair during aortic valve replacement. J Thorac Cardiovasc Surg. 2021;162:44-50.e2.

7. Badhwar V, Rankin JS, He M, Jacobs JP, Furnary AP, Fazzalari FL, et al. Performing concomitant tricuspid valve repair at the time of mitral valve operations is not associated with increased operative mortality. Ann Thorac Surg. 2017;103:587-93.

8. Tang GH, David TE, Singh SK, Maganti MD, Armstrong S, Borger MA. Tricuspid valve repair with an annuloplasty ring results in improved long-term outcomes. Circulation. 2006;114:1577-81.
See Article page 44

\section{Commentary: Tricuspid regurgitation and aortic valve replacement: Act or observe?}

\author{
Siamak Mohammadi, MD, FRCSC, and \\ Dimitri Kalavrouziotis, MD, FRCSC
}

Multivalvular cardiac disease is a highly prevalent condition, for which cardiac surgeons need to have a clear strategy and fine-tune the operative approach to provide the best early and late clinical outcomes for their patients. The management of concomitant tricuspid regurgitation (TR) among patients with mitral valve disease has been fairly well studied, and current guidelines providing direction to the approach to TR in the presence of left-sided valve disease are largely based on data from patients with TR secondary to mitral valve pathology. However, the prevalence and clinical impact of TR, as well as the role of concomitant tricuspid valve (TV) intervention, in patients undergoing surgical aortic valve replacement (AVR) are not completely clear.

In this issue of the Journal, Chancellor and colleagues ${ }^{1}$ retrospectively analyze data from a multicentric cohort of 17,483 adult patients undergoing AVR with or without coronary artery bypass grafting among 19 institutions using the

From the Department of Cardiac Surgery, Quebec Heart and Lung Institute, Quebec City, Quebec, Canada.

Disclosures: Authors have nothing to disclose with regard to commercial support.

Received for publication Feb 27, 2020; accepted for publication Feb 28, 2020; available ahead of print March 9, 2020.

Address for reprints: Siamak Mohammadi, MD, FRCSC, Department of Cardiac Surgery, Quebec Heart and Lung Institute, 2725 Chemin Sainte-Foy, Quebec City, Quebec, Canada, G1V 4G5 (E-mail: siamak.mohammadi@fmed.ulaval.ca).

J Thorac Cardiovasc Surg 2021;162:52-4

$0022-5223 / \$ 36.00$

Copyright (c) 2020 by The American Association for Thoracic Surgery

https://doi.org/10.1016/j.jtcvs.2020.02.097
Check for updates

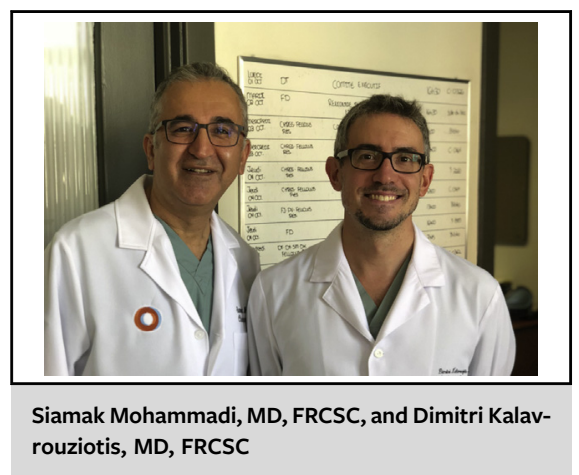

CENTRAL MESSAGE

TR portends a poor outcome after AVR. It remains unclear if correction of the TR improves early and late outcomes in AVR without mitral valve disease.

Society of Thoracic Surgeons database, excluding patients with mitral valve surgery. The prevalence of any TR was $49 \% \quad(\mathrm{n}=8499)$, and the majority of these patients $(85 \%)$ had mild TR. Moderate TR was noted in $6 \%$ of patients $(\mathrm{n}=1060)$, and severe TR was noted in $1 \%$ $(\mathrm{n}=187)$. Only 104 patients with various degrees of TR underwent TV surgery, and strikingly, less than one-third of patients with severe TR $(\mathrm{n}=58)$ underwent TV surgery at the time of AVR. It was not surprising that, in unadjusted comparisons, patients with TR had higher mortality and a higher composite major morbidity rate that was directly proportional to the severity of TR. TV surgery at the time of AVR did not appear to dampen the increased risk associated with TR, because mortality remained higher among those patients with concomitant AVR and TV surgery compared with those with isolated AVR, respectively, 
within all strata of preoperative TR severity: mild ( $15 \%$ vs $3 \%, P=.06)$, moderate $(12 \%$ vs $6 \%, P=.20)$, and severe $(17 \%$ vs $12 \%, P=.38)$. Similar findings were also observed after excluding patients undergoing concomitant coronary artery bypass grafting. Propensity score analysis was used to adjust for the fact that TR was associated with a number of prognosis-limiting variables. After propensity score 1-to-1 matching, in which a little more than half of patients had severe TR, there was a significantly higher rate of composite major morbidity among the AVR + TV surgery group, whereas operative mortality was double in this group compared with AVR without TV surgery $(18 \%$ vs $9 \%)$, although this difference was not statistically significant $(P=.16)$. A major caveat of this analysis was that only $50 \%$ of patients $(\mathrm{n}=55)$ with AVR and TV surgery could be matched, which may have introduced an important selection bias. The authors concluded that preexisting TR was a marker of poor outcomes for patients referred for AVR, but that concomitant TV surgery was not an independent determinant of mortality. They concluded that every attempt should be made to correct TR at the time of AVR, consistent with current peerreviewed guidelines for concomitant TV repair at the time of mitral surgery.

The authors should be congratulated for this multicenter collaborative effort to shed some light on an issue that has hitherto been the subject of single-center observational studies with limited patient numbers. The clinical importance of the study by Chancellor and colleagues ${ }^{1}$ is 2fold: First, the article demonstrates that coexisting TR during AVR is common and associated with increased postoperative morbidity and mortality, and, second, despite a class I recommendation to perform TV surgery at the time of left-sided valve surgery, surgeons are reluctant to perform TV surgery at the time of AVR. Although a large number of patients were recruited and propensity score matching was performed, the authors' recommendation that TR should be corrected during AVR in patients with significant TR cannot be solidly supported by the study's design, and the authors have made a substantial conceptual leap in coming to that conclusion based on their data. The etiology of TR, TV annular size, right ventricular function and secondary remodeling, pulmonary arterial pressure, and indication for TV surgery were not captured because of the retrospective nature of the study. Variables that are not measured cannot be accounted for in multivariate analysis, such as propensity score matching. Unmeasured factors such as TV annular dilation may explain why 20 patients with no or mild TR underwent TV surgery at the time of AVR, but this is not clear. Equally perplexing is the fact that a majority of patients with moderate or greater TR $(n=1143 / 1247,91.6 \%)$ did not undergo TV surgery at the time of AVR. Another major missing piece of the puzzle is the absence of longitudinal data. Late survival and readmission for congestive heart failure, recurrence of TR among patients who received TV repair, and the fate of TR in those patients without TV surgery, all remain extremely relevant unknowns. Following patients with unaddressed TR at the time of AVR is all the more relevant as indications for transcatheter aortic valve replacement (TAVR) continue to expand. Recent data suggest that functional TR in patients with aortic stenosis undergoing TAVR diminishes in severity at both short-term and midterm follow-up post-TAVR in a sizeable proportion of patients, although these data may reflect the systematic exclusion of patients with severe TR and right ventricular dysfunction from consideration for TAVR. ${ }^{2}$

A final word of caution relates to the dismal early clinical outcomes among patients with concomitant TV surgery compared with those who underwent AVR without TV surgery after risk adjustment by propensity score, with double the rate of composite major morbidities $(51 \%$ vs $26 \%)$ and mortality ( $18 \%$ vs $9 \%)$, despite a lack of statistical significance for mortality. These data do not support the recommendation by the authors that significant TR should be addressed at the time of AVR, because TV surgery had no positive impact on early outcomes. The pathophysiologic mechanisms that lead to TR in aortic stenosis in the absence of mitral valve disease are different than those in the setting of mitral valve disease. Functional TR in isolated aortic stenosis may be a surrogate marker of poor outcome to a greater extent than it is in mitral valve disease, due to several factors such as impaired left ventricular diastolic function, although hypotheses remain speculative. Amano and colleagues ${ }^{3}$ recently showed that concomitant TV surgery at the time of AVR did not have an impact on aortic valve-related death or rehospitalization for heart failure, further lending support to the notion that significant TR in aortic stenosis may reflect advanced disease when the prognosis may be unaltered by correcting TR.

The study by Chancellor and colleagues ${ }^{1}$ attempts to address a highly relevant clinical question, is nuanced, and is well conducted. It is a welcome addition to an imperfect body of literature, in which the answer to the question of TR at the time of aortic valve intervention seems to be related to the area of expertise of the clinician performing the study: The surgical AVR literature encourages action, and the TAVR literature encourages observation. Perhaps the greatest value of the study by Chancellor and colleagues ${ }^{1}$ is that it sensitizes the surgical community to the fact that TR among patients undergoing AVR cannot continue to be ignored. Rigorous preoperative evaluation by invasive right-sided pressure monitoring and optimization of right ventricular function before AVR may need to be considered among patients with functional TR. Further studies will be important to identify those patients who are most likely to have a favorable response to TV surgery at the time of AVR. 


\section{References}

1. Chancellor WZ, Mehaffey JH, Beller JP, Hawkins RB, Speir AM, Quader MA, et al. Impact of tricuspid regurgitation with and without repair during aortic valve replacement. J Thorac Cardiovasc Surg. 2021;162:44-50.e2.

2. Sathananthan J, Murdoch DJ, Lindman BR, Zajarias A, Jaber WA, Cremer P, et al. Implications of concomitant tricuspid regurgitation in patients undergoing trans- catheter aortic valve replacement for degenerated surgical aortic bioprosthesis: insights from the PARTNER 2 aortic valve-in-valve registry. JACC Cardiovasc Interv. 2018;11:1154-60.

3. Amano M, Izumi C, Taniguchi T, Morimoto T, Miyake M, Nishimura S, et al, Impact of concomitant tricuspid regurgitation on long-term outcomes in sever aortic stenosis. Eur Heart J Cardiovasc Imaging. 2019;20:353-60.
See Article page 44

\section{Commentary: Tricuspid valve disease at the time of surgical aortic valve replacement: Treat it or leave it?}

\author{
Oliver J. Liakopoulos, MD, ${ }^{\mathrm{a}, \mathrm{b}}$ and \\ Yeong-Hoon Choi, $\mathrm{MD}^{\mathrm{b}}$
}

Current recommendations from guidelines of the American Heart Association/American College of Cardiology and European Society of Cardiology/European Association of Cardiothoracic Surgery reflect the limited evidence from well-controlled trials supporting a more early and aggressive surgical treatment of functional tricuspid regurgitation (TR). ${ }^{1,2}$ Accumulating data from recent observational studies almost uniformly underscore the adverse clinical prognosis of patients with relevant TR and even show that the disease often persists or even progresses after successful correction of mitral or aortic valve disease, including transcatheter aortic valve replacement. ${ }^{3-5}$

Indeed, moderate to severe TR was found to be linked to a substantial increase of mortality in more than 20,000 patients undergoing cardiac surgery over a 24-year follow-up period. ${ }^{6}$ These findings have fired a controversial discussion among experts, and some support a more liberal indication for tricuspid valve surgery (TVS) at the time of left-sided valve surgery. The term "left-sided" valve surgery

\footnotetext{
From the ${ }^{a}$ Department of Cardiothoracic Surgery, Heart Center, University of Cologne, Cologne, Germany; and ${ }^{b}$ Department of Cardiac Surgery, Kerckhoff Clinic Bad Nauheim, Campus Kerckhoff, University of Giessen, Germany.

Disclosures: Authors have nothing to disclose with regard to commercial support.

Received for publication March 2, 2020; accepted for publication March 3, 2020; available ahead of print March 9, 2020.

Address for reprints: Oliver J. Liakopoulos, MD, Department of Cardiac Surgery, Kerckhoff Clinic Bad Nauheim, Campus Kerckhoff, University of Giessen, Germany, Benekestr. 2-8, 61231 Bad Nauheim, Germany (E-mail: oliver@ liakopoulos.de).

J Thorac Cardiovasc Surg 2021;162:54-5

$0022-5223 / \$ 36.00$

Copyright (c) 2020 by The American Association for Thoracic Surgery

https://doi.org/10.1016/j.jtcvs.2020.03.001
}

Check for updates

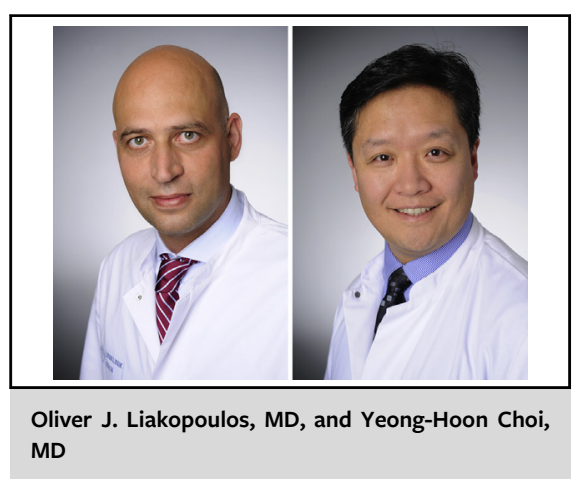

CENTRAL MESSAGE

This retrospective analysis of the

STS database sought to deter-

mine the evidence for a more

aggressive operative strategy for

concomitant tricuspid valve dis-

ease at the time of aortic valve

replacement.

includes 2 different pathophysiologic entities of TR, with mitral valve regurgitation and aortic valve stenosis being the most common. An analysis of the Society of Thoracic Surgeons (STS) database, which included 50,000 patients undergoing TVS between 2000 and 2010, demonstrated that $86 \%$ of tricuspid valve procedures were performed at the time of left-sided valve surgery. ${ }^{7}$ The most common concomitant procedure in the STS database report was mitral valve surgery with approximately $79 \%$ of all cases, whereas aortic valve replacement (AVR) alone at the time of TVS was performed in less than $10 \%$ of all patients. Consequently, current recommendation for TVS are largely based on patient cohorts with concomitant mitral disease and it is unclear whether TR in the presence of aortic disease should be addressed in a similar way., ${ }^{3,6,7}$

In this issue of the Journal, Chancellor and co-workers ${ }^{8}$ report operative outcomes based on a retrospective analysis 